\title{
LA ENSEÑANZA DE LA FILOSOFÍA NATURAL EN LA ÚLTIMA ÉPOCA COLONIAL CHILENA: EL TRATADO ACERCA DE LOS ELEMENTOS Y LAS INSTITUCIONES DE FÍSICA DE MANUEL ANTONIO TALAVERA*
}

\section{The Teaching of Natural Philosophy in the last Chilean Colonial Era: The Treatise on the elements and the Institutiones Physicae by Manuel Antonio Talavera}

\author{
Abel Aravena Zamora \\ Centro de Estudios Avanzados, Universidad de Playa Ancha \\ Centro de Estudios Históricos, Universidad Bernardo O’Higgins
}

\section{Resumen}

El artículo revisa los contenidos generales del Tratado Acerca de los elementos (1792) de Manuel Antonio Talavera, que es parte de los capítulos que estudian la Física especial en su obra Instituciones de Física. En ésta, se combinan las doctrinas de filosofía natural según las enseñanzas de los filósofos modernos (Recentiores), los nuevos datos empíricos generados a partir de los experimentos científicos y la autoridad de las Escrituras. Se intenta mostrar cómo en la enseñanza de la disciplina durante la última época colonial convivieron conocimientos propios de la modernidad matizados con elementos característicos de la escolástica. Presentamos una breve nota biográfica del autor y revisamos desde una perspectiva general los principales aspectos del tratado. La intención es visibilizar el quehacer educativo de Talavera y rescatar parte del legado filosófico latinoamericano.

Palabras clave

Filosofía natural; Historia de la filosofía; Escolástica Americana; Filosofía colonial; Manuel Antonio Talavera.

\footnotetext{
* Este trabajo forma parte del Proyecto Fondecyt de Postdoctorado no 3170144.
} 


\begin{abstract}
This paper examines the general content of the Treatise on the elements (1792) by Manuel Antonio Talavera. This treatise is a part of his work Institutiones Physicae, and is dedicated to the study of Physica particularis. In this work, the doctrine of natural philosophy is combined with new empirical data as well as the authority of the Holy Scriptures. The article analyses how aspects of both modernity and scholasticism coexisted in the teachings of the discipline in the last part of the colonial era. It presents a brief biographical note on the author and from a general perspective examines the principal aspects of the treatise. By examining the principal aspects of the treatise, the article constructs a profile of the educational work of Talavera and highlights legacy of Latin American philosophy.
\end{abstract}

\title{
Keywords
}

Natural Philosophy; History of Philosophy; American Scholastic; Colonial Philosophy; Manuel Antonio Talavera.

\section{Manuel Antonio Talavera: político y docente}

Manuel Antonio Talavera (Villarica, Paraguay 1761 - Mendoza, Argentina 1814) fue un destacado protagonista de las últimas décadas de la época colonial chilena. Pues, por una parte, contamos actualmente con distintos testimonios acerca de su controvertida faceta política, los que incluyen, por ejemplo, el diario ${ }^{1}$ en el que relata detalladamente los sucesos ocurridos en 1810 que dieron origen al proceso de independencia. Por otra parte, se conservan también un par de cursos manuscritos en latín, que dan cuenta de su quehacer como profesor de filosofía en el Santiago de aquella época.

Sobre sus datos biográficos, consta que en 1778 ingresó al colegio de Monserrat de Córdoba del Tucumán (Argentina), en donde obtuvo los grados de Maestro en Artes y el de Doctor en Teología en 1788. Más tarde, en 1790, llegó a Santiago de Chile junto con su tío, Nicolás de Chopitea, y estudió Leyes y Cánones en la Universidad de San Felipe hasta obtener el título de abogado.

Se destacó como un personaje político relevante, pues fue un espíritu firmemente hostil a la revolución y un ferviente partidario del Rey y de la administración española en los territorios americanos. Hacia comienzos de 1810 fue un testigo privilegiado de los primeros síntomas que marcarían el tránsito desde la Colonia hacia la Nación, por lo que juzgó conveniente tomar nota de ellos para construir una base a la historia futura. Así, se transformó en el primer cronista del proceso de independencia. En su diario de memorias, narra detallada y pacientemente los sucesos que presenció entre mayo y octubre del año 1810.

\footnotetext{
${ }^{1}$ Talavera, M.A., Diario, Santiago de Chile, Imprenta Mejía, 1901.
} 
En este sentido, se le considera como una suerte de informante de la actividad política de la colonia, pues, al parecer, comunicaba secretamente al Virrey del Perú las noticias de todos los acontecimientos ocurridos en Chile. De acuerdo con Barros Arana ${ }^{2}$, cuando Talavera vio el desarrollo que tomaba su crónica, decidió continuar para enviarla al Rey, con el propósito de que éste supiera cuánto habían sufrido sus fieles vasallos de Chile por defender los reales derechos.

En esta misma línea, siendo abogado de la Real Audiencia, Talavera jugó un papel importante reuniendo hombres levantados, equipados y pagados por los vecinos ricos, para defender la causa del Rey ${ }^{3}$. Por esta razón, fue tomado prisionero por el ejército revolucionario ${ }^{4}$ y se vio envuelto en diversas dificultades y polémicas. Así, fue mandado a juzgar «por perturbador y atentador contra el sistema y las autoridades revolucionarias constituidas, ordenándosele un castigo ejemplar» ${ }^{5}$.

No obstante, pesar de esta polémica faceta política, Talavera contaba además con otras interesantes cualidades. El historiador Diego Barros Arana lo describe de la siguiente manera:

Era un hombre que en su época en estos países podía llamarse ilustrado, porque conocía las leyes civiles i canónicas i había leído muchos de los comentadores. Padre de una numerosa familia, i sin otra fortuna que su profesión, Talavera era en sus relaciones privadas un modelo de probidad, i como ciudadano, un tipo acabado de fidelidad al rey, de veneración a los gobernadores i de resistencia tenaz a toda innovación política i social' .

Como lo adelanta este testimonio, Manuel Antonio Talavera destacó también como un notable personaje erudito de la época colonial chilena. Sus escritos filosóficos aún inéditos, dan cuenta de que era un hombre culto, que estaba al tanto de las discusiones científicas de su tiempo, en especial sobre aquellas relacionadas con la física experimental expuestas tanto en las célebres Memorias de Trévoux como en diferentes actas de academias científicas europeas, principalmente las de Florencia, París y Londres. Del mismo modo, sus enseñanzas le muestran como un intelectual conocedor de las modernas doctrinas físicas de científicos como Isaac Newton, René Descartes, Pedro Gassendi, Robert Boyle y Jean Antoine Nollet?

\footnotetext{
${ }^{2}$ Barros Arana, D., Historia Jeneral de Chile, tomo VIII, Santiago de Chile, Rafael Jover Editor, 1887, p. 481.

${ }^{3}$ Martínez, M., Memoria histórica sobre la revolución de Chile, desde el cautiverio de Fernando VII, hasta 1814, Valparaíso, Imprenta Europea, 1848, p. 56.

${ }^{4}$ Ibid., p. 93.

${ }^{5}$ Ibid., pp. 131, 350, 373; Feliú Cruz, G., Vida de don Manuel Antonio Talavera: primer cronista de la Revolución de la Independencia de Chile, Santiago, Impr. Cóndor, 1937, p. 95.

${ }^{6}$ Barros Arana, Historia Jeneral de Chile, op. cit., p. 480.

${ }^{7}$ Cf. Jacksić, I., Rebeldes Académicos. La filosofía chilena desde la Independencia hasta 1989, Santiago de Chile, Ediciones Universidad Diego Portales, 2013, p. 43; Infante Martín, J., «Curas, frailes y otros alborotadores en la independencia de Chile», Revista de Estudios Histórico-Jurídicos, 37 (2015), pp. 503-520, en p. 512.
} 
Talavera se desempeñó entre los años 1791 y 1792 como profesor de Artes en el Colegio San Carlos de Santiago de Chile ${ }^{8}$ y, más tarde, en 1794, ejerció en la misma ciudad como profesor particular de filosofía, regentando un estudio al que acudían los estudiantes más distinguidos de la sociedad de aquel tiempo9. Contaba entonces con ocho alumnos para el primer curso y con siete para el tercero, de modo que más de la mitad de todos los estudiantes seculares que entonces se educaban en Santiago de Chile acudían a su estudio ${ }^{10}$.

Fruto de su docencia en el Colegio San Carlos, se conservan actualmente dos partes del curso filosófico del trienio 1791-1793. La primera parte corresponde a algunos fragmentos del curso de ética, fechado en marzo de 1791, mientras que la segunda, a las Institutiones de Física y datan del año 1792. Ambos cursos están escritos en latín, según la usanza de la época, y fueron copiados por el mismo Talavera para desarrollar sus labores docentes. Se conservaría también, de acuerdo con Medina ${ }^{11}$, una obra titulada Tractactus scholasticus De voluntate Dei, de la que actualmente no tenemos ninguna otra noticia.

Del primer manuscrito mencionado se conservan solamente cinco fojas en el Archivo Jesuita del Colegio San Ignacio ${ }^{12}$ de Santiago de Chile. La traducción castellana de su extenso título es Instituciones de Ética del curso trienal de Filosofía o Filosofía Moral, dispuestas y elucubradas para los nobles alumnos del Colegio de San Carlos de la Ciudad de Santiago del Reino de Chile, con método claro, por el señor Manuel Antonio Talavera, Maestro Laureado de la Real Universidad de Córdoba en Filosofía, en otro tiempo alumno del Colegio de Monserrat, y ahora en el mismo Colegio de San Carlos profesor de la Cátedra de Artes. Empezadas el 10 de Marzo del año del Señor de $1791^{13}$.

${ }^{8}$ Díaz, G., Hombres y documentos de la filosofía española, VII, Madrid, Consejo Superior de Investigaciones Científicas, 2003, 446; Hanisch, W., En torno a la Filosofía en Chile (1594-1810), Santiago de Chile, Ediciones Historia, Universidad Católica de Chile, 1963, p. 102; Furlong, G., Nacimiento y desarrollo de la filosofía en el Río de la Plata, Buenos Aires, Ed. Kraft, 1952, p. 383; Frontaura, J.M., Historia del Convictorio Carolino, Santiago de Chile, Imprenta Nacional, 1889, pp. 3-33; Barros Arana, Historia Jeneral de Chile, op. cit., p. 480.

${ }^{9}$ Medina, J.T., Historia de la Real Universidad de San Felipe de Santiago de Chile, tomo I, Santiago de Chile, Soc. Imp. Y Lit. Universo, 1928, p. 562.

${ }^{10}$ Aquellos ilustres discípulos de Talavera fueron Francisco de Paula Ramírez, Timoteo Arratia, Pedro Botarro, Diego Lavaqui, Ramón Pedregal, Agustín Palazuelos, Juan Alberto Mardo-nes, Manuel Infante, Marcial Lavaqui, Manuel Luján, Joaquín Fernández, Bernardo Maceira, José Miguel Infante, José Antonio Pedregal y Manuel Mascayano. Cf. Medina, Historia de la Real Universidad, op. cit., p. 562.

${ }^{11}$ Medina, J. T., La instrucción pública en Chile desde sus orígenes hasta la fundación de la Universidad de San Felipe, Santiago de Chile, Imprenta Elzeviriana, 1905, p. CCCLXII.

${ }^{12}$ Con el nombre de Tratado Filosofía Talavera. 2/ I/ 311 Carp. 08.

${ }^{13}$ Trienalis Philosofici Cursus Institutiones Ethicae, sive Philosophia Moralis Nobilibus Sancti Caroli Collegii Jacobopolitana Urbis Regni Chilensis Alumnis clara methodo digestae ac elucubratae a Domino Emmanuele Antonio de Thalavera Regali Cordubensi Academia Laureato in Philosofia Magistro, olim Colegii Monserratensis Alumno, nunc vero in eodem S. C. Collegio Artium Cathedrae Moderatore. Inceptae die 10 
Por otro lado, el segundo manuscrito con las enseñanzas de Talavera se conserva actualmente en el Archivo Central Andrés Bello de la Universidad de Chile. Su título traducido al castellano es Instituciones de Física del curso trienal de Filosofía, segunda parte de la filosofía natural, es decir, lo que comprende la Física particular. Según las más verdaderas enseñanzas e indudables experimentos de los filósofos recientes, dispuestas y elucubradas para los nobles alumnos del Colegio de San Carlos de la Ciudad de Santiago del Reino de Chile, con método claro, por el señor Manuel Antonio Talavera, Maestro Laureado de la Real Universidad de Córdoba en Filosofía, en otro tiempo alumno del Colegio de Monserrat, y ahora en el mismo Colegio de San Carlos profesor de la Cátedra de Artes. Empezadas el 27 de Mayo del año del Señor de $1792^{14}$.

\section{Las Instituciones de Física y el Tratado acerca de los elementos}

Las Instituciones de Física son parte del curso trienal de filosofía (1791-1793) de Manuel Antonio Talavera. En ellas, expone las doctrinas de filosofía natural según las enseñanzas de los filósofos modernos, llamados también Recentiores, y los nuevos datos empíricos generados a partir de los numerosos experimentos físicos realizados por los científicos de vanguardia de la época. Vale la pena mencionar que, ya en el propio título de la obra, el autor las describe como «verdaderas enseñanzas e indudables experimentos».

Actualmente sólo se conserva la parte dedicada al estudio de la Física especial, mientras que del tratado que aborda la Física general, al que el mismo Talavera hace numerosas referencias, no conocemos su paradero. La Física especial se divide, a su vez, en tres tratados: el primero versa sobre los elementos (De elementis, ff. 3r-111r), el se-gundo trata acerca de los seres vivos (De viventibus, ff. 112r-179r) y el último aborda el estudio de los cuerpos celestes (De coelo et Mundo, ff. 179r-212v).

Un rasgo característico de este curso, en el que quisiéramos centrar fundamentalmente nuestra atención, es el eclecticismo de sus enseñanzas, lo que a juicio de Celina Lértora Mendoza es, justamente, propio del período pre-ilustrado de la escolástica americana. Pues, por un lado, contiene numerosas referencias a diversas teorías de científicos modernos, a las observaciones y experimentos físicos reseñados en las famosas $\mathrm{Me}$ morias de Trévoux y también a los diferentes boletines divulgativos de las Academias de Ciencias de Florencia, París y Londres ${ }^{15}$. Y, por otro lado, a lo largo de la obra Talavera

mensis Martii anii Domini 1791. Corregimos el error de Díaz, Hombres y documentos, op. cit., p. 446 y de Hanisch, En torno a la filosofía, op. cit., p. 102, quienes fechaban la obra en 1795.

${ }_{14}$ Trienalis Philosofici Cursus Institutiones Physicae, secundam naturalis Phylosophiae partem Physicam scilicet particularem complectentes. Iuxta veriora Recentiorum placita illorumque inconcussa experimenta. Nobilibus Sancti Caroli Collegii Jacobopolitana Urbis Regni Chilensis Alumnis clara methodo digestae ac elucubratae a Domino Emmanuele Antonio de Talavera Regali Cordubensi Academia Laureato in Philosofia Magistro, olim Colegii Monserratensis Alumno, nunc vero in eodem S. Caroli Collegio Artium Cathedrae Moderatore. Inceptae die Tertio Nomas Maii anii Domini 1792, 213ff.

${ }^{15}$ Cf. Lértora Mendoza, C., «La Translatio Studiorum y la Scholastica Americana. Un tema actual», Revista Española de Filosofía Medieval, 25 (2018), pp. 81-96; Lértora Mendoza, C., La enseñanza 
recurre frecuentemente a la autoridad y enseñanza simplificada de distintos filósofos clásicos y medievales y, en menor medida, a algunos pasajes de las Escrituras ${ }^{16}$.

Asimismo, un valor agregado de las Instituciones de Física es que constituyen un documento original de manos del propio profesor, lo que es poco frecuente en los códices coloniales que se conservan hasta nuestros días ${ }^{17}$. En efecto, la gran mayoría de éstos han sido copiados por alumnos al dictado del profesor -con todas las limitantes que ello supone- $y$ tienen una finalidad eminentemente didáctica ${ }^{18}$.

Por ello, con la finalidad de conocer y valorar el quehacer filosófico de Manuel Antonio Talavera, especialmente su enseñanza de la filosofía natural, revisaremos los aspectos más destacables del Tratado acerca de los elementos a la luz del contexto filosófico de la época. Intentaremos exponer, en líneas generales, los principales temas de estudio, así como los métodos de argumentación y prueba de las diferentes enseñanzas. Por la misma razón, cuando nos ha sido posible, indicamos una breve información orientativa sobre los autores y obras estudiadas en el tratado.

De acuerdo con lo anterior, pretendemos rescatar y visibilizar esta fuente inédita de la enseñanza de la filosofía en el Chile de la última época colonial, y, consecuentemente, posibilitar futuras investigaciones filosóficas, educativas e históricas.

\section{El Tratado acerca de los elementos}

Luego de estudiar en la Física general acerca de los principios universales, las causas, las propiedades y las afecciones de las cosas, Talavera centra su estudio en el origen de las singularidades de cada cuerpo ${ }^{19}$.

Así, para iniciar el curso dedicado a la Física especial, el preceptor aclara que aunque el término elemento se entienda de muchas maneras en las disciplinas científicas, los

de la filosofía en tiempos de la Colonia: análisis de cursos manuscritos, Buenos Aires, Fundación para la Educación, la Ciencia y la Cultura, 1979, pp. 11-24.

${ }^{16} \mathrm{Cf}$. Aravena Zamora, A., «Sobre unas conclusiones filosóficas defendidas en la Real Universidad de San Felipe (Santiago de Chile, 1807)», Revista Communitas, 3/6 (2019), pp. 220-243; Aravena Zamora, A., «Filosofía en el Chile colonial: el curso de animástica de fray Juan de Fuica», Anales del Seminario de Historia de la Filosofía, 33/2 (2016), pp. 478-486; Aravena Zamora, A., «Un curso de filosofía del Chile colonial: los Comentarios Acerca del alma de fray Juan de Fuica (1689)», Veritas, 35 (2016), pp. 84-96.

${ }^{17} \mathrm{Cf}$. Aravena Zamora, A., «Manuscritos filosóficos coloniales conservados en el Archivo Nacional Histórico de Santiago de Chile», Revista Española de Filosofía Medieval, 22 (2015), pp. 289-305; Aravena Zamora, A., «Una contribución al corpus filosófico colonial chileno: los cursos de animástica», Atenea, 519 (2019), pp. 11-29.

${ }^{18}$ Cf. Lértora Mendoza, C., «La Translatio Studiorum», op. cit., pp. 91-92; Lértora Mendoza, La enseñanza de la filosofía, op. cit., pp. 24-30.

19 Talavera, M.A., Trienalis Philosofici Cursus Institutiones Physicae, secundam naturalis Phylosophiae partem Physicam scilicet particularem complectentes, Ms., 1792, f. 1r. 
filósofos de la naturaleza - los Physicos- lo conciben como algún cuerpo simple, a partir del que los cuerpos se componen y en el que todos se disuelven. De allí que los cuerpos sean denominados un compuesto mixto de los mismos elementos. Tal compuesto, advierte, puede ser de dos clases: imperfecto, si la unión de los elementos puede disolverse; o perfecto, si, por el contrario, dicha unión persevera ${ }^{20}$.

Aclara que acerca del número de los elementos son muchas las opiniones entre los filósofos antiguos y modernos, por lo que se ocupará, antes que todo, en definir qué y cuántos son los elementos. Al respecto, explica que algunos físicos postulan que sólo hay tres elementos, mientras otros, cuatro o cinco, pero que todos sus argumentos son similares. Para él, de acuerdo con lo que aparece a los sentidos, los elementos son cuatro: tierra, agua, aire y fuego. Por ello, dedicará un capítulo especial al estudio de cada uno de ellos y, en consecuencia, estructura su tratado en igual número de capítulos.

No obstante, antes de seguir, Talavera aclara lo que no debe entenderse por elemento en este curso de física especial. Sobre ello, escribe:

Entonces, el concepto es que el fuego vulgar, el agua corriente, el aire que respiramos y la tierra que pisamos, no son en verdad los elementos propiamente dichos, sin embargo pueden llamarse de ese modo impropiamente hablando, porque es evidente que una mezcla de muchos de ellos constituye los cuerpos sensibles ${ }^{21}$.

Luego de afirmar, «junto con el consenso unánime de los filósofos», que todos los cuerpos son afectados por la gravedad y atraídos hacia el centro de la tierra, el autor pregunta si los elementos gravitan en su lugar propio respectivo. Vale decir, si todos los elementos se dirigen hacia un único centro de gravedad, el centro indivisible de la tierra al que todos los cuerpos tienden; o si tienden hacia su lugar respectivo, aquel que ocupan los cuerpos dependiendo de su mayor o menor peso o levedad. En esto, Talavera indica que los peripatéticos sostenían, a través de argumentos y experimentos, que los elementos no gravitan en su lugar propio respectivo, lo que está en contra de la opinión de los filósofos modernos ${ }^{22}$.

Para esta cuestión preliminar, expone dos conclusiones, que afirman que los elementos gravitan en lugar propio, y resuelve luego cuatro argumentos. En el desarrollo de éstos presenta numerosa evidencia empírica, pues, por ejemplo, hace referencia a las observaciones del doctor parisino Joseph Pitton de Tournefort (1656-1708) sobre el efecto de la gravedad en las plantas, expuestas en el tomo IV (1702) de las Mémoires de Trévoux; y también ofrece las observaciones del Padre De Lana (1633-1687), en su obra

${ }^{20}$ Ibid., f. 3r. Cf. Aravena Zamora, A., «La Física contra Tomistas de Lorenzo Romo S. J.: apuntes sobre una controversia filosófica en el Chile del siglo XVIII», Universum, 33/2 (2018), pp. 53-73.

${ }^{21}$ Ibid., ff. 3v-4r.

${ }^{22}$ Ibid., f. $4 \mathrm{v}$. 
Magisterium naturae et artis, sobre la influencia de la gravedad en el agua y su efecto en los buzos ${ }^{23}$.

Vale la pena señalar que ambas informaciones las encontramos en una referencia exacta de la Physica particularis (1760), obra del jesuita Berthold Hauser (1713-1762) muy utilizada por los profesores de la época colonial en América del Sur. Hallamos en este punto, además, varias referencias sobre estática expuestas en el Cursus seu Mundus Mathematicus (1674) del jesuita francés Milliet Dechales (1621-1678); y también numerosas menciones a distintos pasajes de la Física General del propio Talavera, obra que como hemos indicado antes- lamentablemente no se nos ha conservado.

\section{Sobre el Fuego}

En este primer capítulo, que se estructura en cinco artículos que contienen a su vez un número variables de cuestiones, Talavera analiza primero las diferentes doctrinas sobre el fuego (ignis). Inicialmente, aborda el estudio del fuego elemental (Sulphur) y sus propiedades, para lo que se vale de las enseñanzas de autores como Aristóteles, Descartes e Isaac Newton.

Explica que, según el Estagirita, el fuego es un elemento caliente y seco (Meteora, I, 3), lo que también suscriben los peripatéticos. Para los cartesianos, en cambio, el fuego es un cuerpo mixto liquidísimo y sutilísimo como ningún otro; mientras que muchos newtonianos suponen que el fuego sublunar no es una substancia peculiar, sino sólo fermentaciones de partes sulfurosas y movimiento rapidísimo. Por ello, en las sentencias de estos autores, las partes sulfurosas se distinguen del fuego sólo por el movimiento ${ }^{24}$.

De acuerdo con esta conclusión, el fuego elemental es una materia etérea o una sutilísima substancia corpórea. Así, la movilidad, sutileza y agilidad del fuego elemental lo asemejan a las propiedades que los físicos suponen al éter. En este punto, Talavera fundamenta su enseñanza en dos obras de Benito Jerónimo Feijoo (1676-1764), específicamente nombra las Cartas eruditas y curiosas y el Teatro crítico universal, y también remite a un pasaje estudiado en su propia obra Física General.

Posteriormente, pregunta si por medio de un arte humano pueden producirse luces que ardan perpetuamente, inquietud que compartían -dice- los filósofos antiguos y también muchos modernos, entre ellos el mismo Feijoo y Hauser. A partir de las informaciones entregadas por éste último, Talavera concluye que, considerando los principios físicos del ingenio humano, es del todo imposible tal artificio ${ }^{25}$.

\footnotetext{
${ }^{23}$ Ibid., ff. 4v-6v.

${ }^{24}$ Ibid., ff. 6v-7r.

${ }^{25}$ Ibid., ff. 8v-10v.
} 
Luego, expone ciertas paradojas del efecto del fuego, para lo que nuevamente recurre a diversos datos de observaciones astronómicas y experimentos ópticos comentados por autores como Pierre Bouguer (1698-1758), en su obra Ignis Mathematicus, y Bernard le Bovier de Fontenelle (1657-1757), en Conversaciones acerca de la pluralidad de mundos (1686), además de los ya mencionados ${ }^{26}$. Más adelante, Talavera se dedica a estudiar el fuego subterráneo, indicando que ningún físico ignora que en las entrañas de la tierra se halla fuego, cuya prueba son los terremotos, las termas y los volcanes. Sobre estos últimos, añade que son célebres el Etna y el Vesubio, y los que se encuentran en las Islas Canarias, en Filipinas, en las Islas Molucas, en Perú y también en Chile. Para reforzar mayormente su argumentación, relata además distintos episodios de erupciones volcánicas (por ejemplo, en Catania, Congo e Inglaterra) y de terremotos, valiéndose de obras como Recreación Filosófica o Diálogo sobre la Filosofía Natural (1785) de Teodoro de Almeida (1722-1804) y Mundus Subterraneus (1665) de Athanasius Kircher (1601-1680), aunque también recurre a los testimonios de los antiguos Plinio y Estrabón ${ }^{27}$.

En cuanto al fenómeno del fuego artificial, Talavera comenta que su desarrollo ha estado ligado tanto a fines festivos como bélicos. Para explicarlo, relata la anécdota del químico y franciscano alemán Bertoldo Schuber, quien experimentó tempranamente con pólvora, y rescata también el testimonio de Feijoo en el Teatro Crítico Universal acerca de su detonación. Además, describe con detalle distintos experimentos que demuestran el control artificial del fuego (por ejemplo, su dirección controlada en la guerra). En este contexto, destaca los testimonios de Pedro van Musschenbroek (16921761), Jean Antoine Nollet (1700-1770) y los recogidos a su vez en las obras de Hauser y Almeida ${ }^{28}$.

Por otra parte, el preceptor analiza también las doctrinas referentes a los fenómenos del relámpago, del rayo y del trueno, que, para el género humano, son -explicaterribles y formidables a la vez. Primero, estudia la naturaleza y formación del relámpago (fulgur), expone las diferentes interacciones físico-químicas que lo causan y concluye que es una exhalación sulfuro-nitrosa al interior de una nube inflamada, que se desvanece en el aire. Para probarlo, refiere el ameno testimonio de Feijoo sobre un intenso fenómeno de esta naturaleza, ocurrido en la ciudad de Oviedo el año 1730. Luego, en una cuestión exclusiva, desarrolla largamente las teorías acerca del lugar en dónde se origina el relámpago y fundamenta sus argumentos en las enseñanzas de autores como Gassendi, Musschenbroek, Feijoo y otros Recentiores ${ }^{29}$.

Después, Talavera expone diferentes doctrinas sobre el rayo (fulmen), al que define como una exhalación sulfuro-nitrosa más fructífera, más espesa y más densa que la materia del relámpago, inflamada y movida por una vibración muy fuerte. Seguidamente, se enfoca en la experiencia concreta y describe qué es lo que ocurre cuando un rayo cae

\footnotetext{
${ }^{26}$ Ibid., ff. $10 \mathrm{v}-11 \mathrm{v}$.

${ }^{27}$ Ibid., ff. $12 \mathrm{r}-13 \mathrm{v}$.

${ }^{28}$ Ibid., ff. $13 \mathrm{v}-15 \mathrm{v}$.

${ }^{29}$ Ibid., ff. $15 \mathrm{v}-17 \mathrm{v}$.
} 
en los árboles, en las piedras e incluso en la piel, brindando distintas anécdotas y detallados testimonios al respecto ${ }^{30}$.

Más adelante, en corolarios especiales, explica algunas similitudes de los efectos del rayo con la electricidad, según -dice- consta en la experiencia. En esta misma línea, desarrolla largamente cuáles son los lugares más y menos peligros para la caída de rayos y dedica no pocos pasajes a referirse a los edificios, las campanas, las cúspides metálicas y otros conductores de la electricidad. Entrega asimismo remedios para protegerse de los rayos e indica que el pararrayos (vectis ferrea) es el instrumento más eficaz. Además, explica las ideas y experimentos de Benjamín Franklin y se refiere a los peligros que se corren al experimentar con la fuerza eléctrica. Al respecto, comenta la anécdota del accidente que le costó la vida al físico alemán George Wilhelm Richmann (1711-1753), de la Academia de Ciencias de San Petersburgo, quien, al estudiar la electricidad atmosférica, falleció electrocutado por una descarga mientras trataba de reproducir los experimentos de Franklin con la cometa ${ }^{31}$.

Desde un punto de vista metodológico, es destacable que en este contexto, Talavera recomiende, a quien esté interesado y quiera saber más sobre la electricidad atmosférica, leer con mayor cuidado la parte dedicada a la física general en las Institutiones Philosophicae de Joseph Valla y también las obras de José Aignan Sigaud Lafond (1730-1810) ${ }^{32}$.

Luego, hallamos otras muchas descripciones relacionadas con el tema en estudio. Así, encontramos el relato de numerosas anécdotas sobre los globos ignotos, que eran capaces de lanzar pólvora compactada, y se refiere también a los fenómenos atmosféricos que permiten la formación de halos o coronas alrededor del sol y de la luna. En este punto, explica las observaciones empíricas de Musschenbroek y de Robert Boyle (16271691) en la Physica mechanica (1660) ${ }^{33}$.

Después, el preceptor estudia acerca de la caída de estrellas y de los fuegos fatuos, llamados también ambulones. Sobre estos últimos, descarta la opinión vulgar de que sean almas errantes, en pena durante siglos. Y en cuanto a las estrellas, recuerda las denominaciones de Castor, Pólux y Elena de la mitología griega para referirse a la constelación de Géminis, estrellas que servían de guías a los navegantes ${ }^{34}$.

Al referirse al fenómeno del trueno (tonitruum), Talavera explica que son variadas las opiniones de los filósofos sobre sus causas. Algunos dicen que el trueno consiste en una violenta fracción de una nube, causada por el ascenso de materia inflamable en su seno. Descartes y otros afirman que el trueno es causado por la colisión de nubes, en donde la nube superior cae con enorme fuerza sobre la inferior. Sin embargo, -dicetales opiniones de los filósofos, si bien admiten cierta verdad, presentan también otras

\footnotetext{
${ }^{30}$ Ibid., ff. $17 \mathrm{v}-18 \mathrm{v}$.

${ }^{31} \mathrm{Ibid}$., ff. 18v-21v.

${ }^{32}$ Ibid., f. 21v.

${ }^{33}$ Ibid., ff. 21v-23r.

${ }^{34}$ Ibid., ff. 23r-24r.
} 
muchas dificultades. Por ello, define el trueno como el sonido causado por una fuertísima división o vibración del aire, originado por una súbita expansión de materia inflamable en las nubes. Como prueba de su argumentación, refiere las observaciones de Musschenbroek en la obra Elementa physicae, y los experimentos de la Academia de las Ciencias de 1699 descritos por Feijoo y Regnault ${ }^{35}$.

Por último, para cerrar el capítulo, Talavera, primero, propone y revisa los fundamentos de las opiniones contrarias con respecto al origen de los rayos ${ }^{36} \mathrm{y}$, luego, explica con detención algunos otros fenómenos del fuego, fundamentalmente aquellos producidos por su interacción con los elementos aire y agua ${ }^{37}$.

\section{Sobre el Aire}

El segundo capítulo del Tratado acerca de los elementos aborda el estudio del elemento aire (aer). Los contenidos estudiados se estructuran en cuatro cuestiones y la exposición se desarrolla con diferentes grados de profundidad y extensión.

En la primera cuestión, Talavera se refiere a las propiedades generales del aire y explica los conceptos de gravedad y elasticidad. Luego de distinguir dos géneros de aire (la atmósfera que circunda a la tierra y el aire puro) y de referirse brevemente a sus tres distintas regiones, advierte que sobre la naturaleza de este elemento, la opinión de los filósofos no es una. De acuerdo con esto enseña que «los Peripatéticos pensaban que Aristóteles sostenía que el aire es un elemento cálido y húmedo ${ }^{38}$, mientras que para otros la naturaleza de este elemento parecía ser más bien fría. En cambio, según algunos Recentiores el aire que respiramos es vapor y una acumulación de exhalaciones y, para otros, se compone de partículas propias y otras comunes a los vapores y exhalaciones. Una vez planteado esto, el preceptor analiza diversas proposiciones sobre la naturaleza, elasticidad, gravedad y levedad del aire ${ }^{39}$.

En la segunda cuestión, Talavera expone largamente las discusiones en torno a si el ascenso de los líquidos se produce por la gravedad y elasticidad del aire o, más bien, por la antigua doctrina del horror al vacío (horror vacui). Grosso modo, según la interpretación escolástica de las ideas aristotélicas, que por cierto buscaba ajustarlas a los dogmas del cristianismo, la naturaleza aborrece el vacío. Sin embargo, determinados fenómenos observados en diversos experimentos no podían explicarse satisfactoriamente por medio de aquella teoría.

Por su parte, Talavera describe someramente los experimentos de Evangelista Torricelli, quien demostró la falsedad de la teoría sustentada por los peripatéticos $\mathrm{y}$,

\footnotetext{
${ }^{35}$ Ibid., ff. 24r-26r.

${ }^{36} \mathrm{Ibid}$., ff. $28 \mathrm{r}-29 \mathrm{r}$.

${ }^{37}$ Ibid., ff. 29r-30v.

${ }^{38}$ Ibid., f. $31 \mathrm{v}$.

${ }^{39}$ Ibid., ff. 31v-33v.
} 
consecuentemente, probó la existencia del vacío y la presión del aire. En la conclusión de la cuestión, que según dice es común a todos los Recentiores, el preceptor escribe:

El ascenso y la suspensión de los líquidos en los tubos vulgares y en las bombas dependen de la gravedad del aire y el elaterio, y los admirables efectos que se presentan, que para los Peripatéticos se adscriben al miedo al vacío, deben adaptarse a la presión del aire $[. . .]^{40}$.

En esta misma línea, comenta los aportes empíricos de Pascal, del Padre Lana y de Musschenbroek, de nuevo a partir de la exposición de Hauser en la Physica particularis, y describe también brevemente el experimento de Otto von Guericke, conocido como «experimento de Magdeburgo», que demostraba los efectos del vacío. Asimismo, expone con detalle los diversos estudios de Boyle, en la mencionada Physica mechanica, acerca de la elasticidad y la gravedad del aire ${ }^{41}$.

Desde un punto de vista metodológico, hallamos nuevamente una característica de la enseñanza de Talavera a lo largo del curso, pues revisa y comenta las ideas de diversos autores, entregando la referencia exacta de sus obras. Así, al estudiar los diferentes aspectos relacionados con la atmósfera destaca las observaciones de estática de Dechales, de óptica de Newton y de aerometría de Christian Wolff (1679-1754), así como los recurrentes testimonios de Hauser. En esta línea, el mismo Talavera dice que apoya su argumentación en un texto de la Historia de la Academia de las Ciencias de 1740, titulado Mecánica sobre el efecto de las bombas en el vacío. Asimismo, para saber más sobre este tema, recomienda nuevamente consultar las obras de Nollet, Sigaud Lafond, Almeida y del «clarísimo» Hauser ${ }^{42}$.

Más adelante, el preceptor interroga sobre la procedencia del ascenso de los líquidos en los tubos capilares. En ellos, -dice- los fluidos se conducen muy estrechamente a través de una sección circular y reciben su nombre por la similitud con el espesor del cabello humano. En este tema, la metodología de estudio adoptada es la misma. Primero, expone detalladamente nueve opiniones sobre los principales fenómenos observados en los tubos capilares y, luego, rechaza tres sentencias (la cartesiana, la de muchos físicos experimentales y otra de la que no menciona autor) respecto a la causa del ascenso de los líquidos en los tubos. Por último, para refutarlas, comenta los experimentos de G. B. Bilfinger (1693-1750), los testimonios de Nollet y, los del Padre Gendil, respecto a la experimentación con tubos capilares de oro. De acuerdo con Talavera, todos estos testimonios confirmarían el papel causal de la presión del aire y el elaterio en el ascenso de los líquidos en los tubos capilares ${ }^{43}$.

Con la finalidad de aclarar por completo y demostrar las causas del fenómeno en estudio, en el escolio de esta cuestión Talavera desarrolla algunas disquisiciones físicas.

\footnotetext{
${ }^{40}$ Ibid., f. 34 r.

${ }^{41}$ Ibid., f. $34 \mathrm{v}$.

${ }^{42}$ Ibid., ff. $37 \mathrm{r}-40 \mathrm{v}$.

${ }^{43}$ Ibid., ff. 40v-43v.
} 
Así, destaca las similitudes entre el fenómeno observado en los tubos capilares y el observado en los vegetales, pues en las plantas los líquidos nutrientes van desde la tierra al tronco, luego a las ramas y finalmente a las hojas. En todo caso, indica que para conseguir mayor información sobre estos fenómenos pueden consultarse también las obras de los «clarísimos» Hauser, Almeida y Emmanuel Monteyro ${ }^{44}$.

Luego, se refiere a los resultados de algunos experimentos con el aire efectuados sin una máquina neumática ${ }^{45}$. Aquí, describe y explica dos experimentos sobre la presión del aire: uno con un tubo de Torricelli y el otro con un tubo vítreo, de los que incluye también algunas ilustraciones explicativas, como la que adjuntamos a continuación.

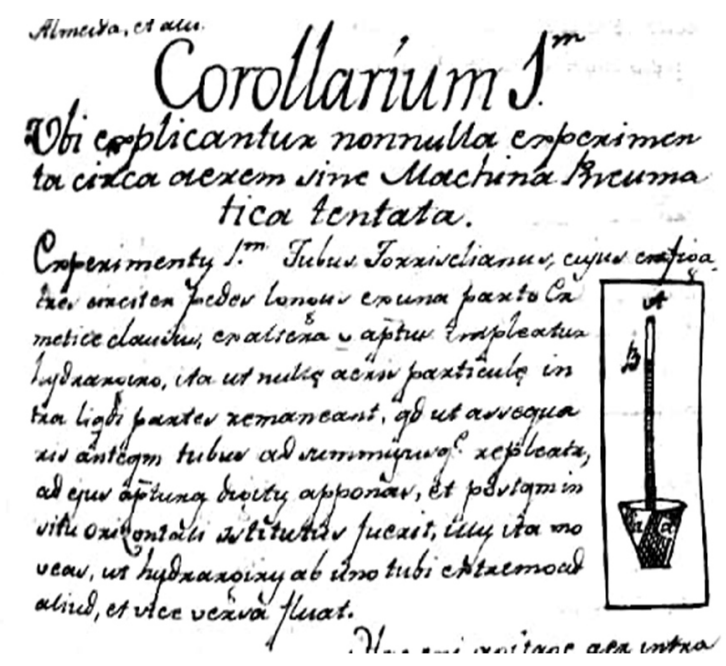

Detalle, f. 44r.

Después, en otro corolario, mediante la descripción de otros seis experimentos afines, Talavera explica el funcionamiento de la máquina neumática y, con ello, -dicequeda demostrada la elasticidad del aire ${ }^{46}$.

\footnotetext{
${ }^{44}$ Ibid., ff. 43v-44r.

${ }^{45}$ Ibid., ff. 44r-46v.

${ }^{46}$ Ibid., ff. 46v-48r.
} 
Posteriormente, en el tercer y último corolario, define, explica e ilustra el funcionamiento de los instrumentos para estimar el peso, el calor, la densidad y la humedad del aire. Así, como puede verse en la imagen que sigue, se refiere al barómetro, al termómetro, al manómetro y al higrómetro, respectivamente. Aquí, hallamos que el preceptor transcribe, al pie de la letra, el texto completo de la Physica generalis de Joseph Valla (Dissert. VI, sectio IV) ${ }^{47}$.

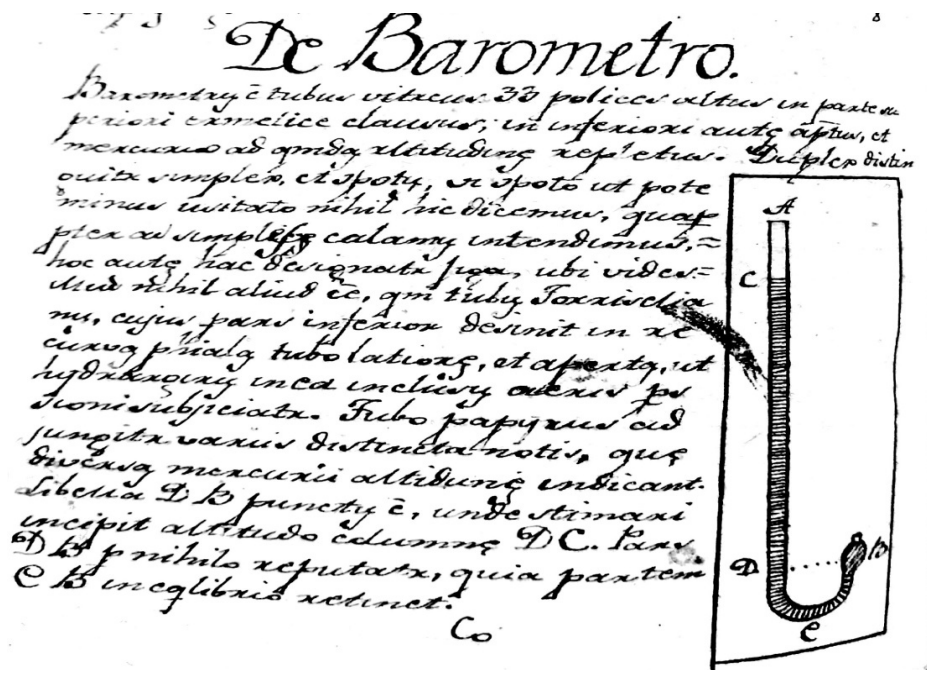

Detalle, f. $48 \mathrm{v}$.

En la última cuestión de este capítulo, Talavera estudia el fenómeno de los vientos. Para ello, plantea inicialmente distintas cuestiones: de dónde proceden, cuáles son sus causas y cuáles sus principales especies. Luego, presenta dos conclusiones explicativas. La primera indica que «la causa próxima e inmediata de los vientos es el elevado equilibrio de los trechos de los aires»; mientras que la segunda afirma que «las causas remotas de los vientos son muchas, pero particularmente el calor del sol, y el fuego subterráneo, la elasticidad del aire, el descenso de las nubes, las exhalaciones secas, etcétera $»^{48}$. Seguidamente, el preceptor explica cada una de las causas indicadas, aduciendo siempre pruebas según consta en la experiencia.

Al respecto, agrega que otra causa de los vientos podría ser los diversos géneros de vapores y hálitos que se fermentan en la atmósfera y se refiere al papel de los diferentes procesos de rarefacción y calefacción en la producción de los vientos. En este punto, explica las causas de los huracanes, recurriendo principalmente a las enseñanzas de Musschenbroek, y distingue tres especies de vientos: egnefias, tifón (turbo) y huracán

\footnotetext{
${ }^{47}$ Ibid., ff. 48v-50v.

${ }^{48}$ Ibid., f. 51r.
} 
(praester). Después, el preceptor explica las diferentes utilidades y propiedades de los vientos ${ }^{49}$.

Como hemos señalado, a lo largo del tratado Talavera ofrece diversos testimonios que describen empíricamente el fenómeno particular en estudio. En este contexto, para ilustrar las intensas consecuencias de los vientos, relata una anécdota histórica sin mencionar su fuente:

En el año 1740, en el Mar del Sur quedó inmersa de agua la nave española de nombre La Hermiona, la que intentaba superar, contra los cuatro vientos a la vez, el promontorio austral, vulgarmente conocido como el Cabo de Hornos, bajo la dirección de Don José Pizarro, de lo que los testimonios son muchos, tanto en esta ciudad como en Buenos Aires $[\ldots]^{50}$.

En esta misma línea, se halla el intento de explicar científicamente circunstancias locales, referidas específicamente en este caso a un determinado periodo de intensos vientos en la ciudad de Santiago de Chile. Así, Talavera se esfuerza en dar cuenta del fenómeno de fuertes y frecuentes vientos, que -según dice- se presenta en la ciudad entre los meses de agosto y septiembre de cada año. De acuerdo con el preceptor, se producen porque, en esos meses, el sol viene desde el norte al sur, lo que rarefacciona tanto el aire intermedio como el ascenso de las exhalaciones, y lo más perceptible es el derretimiento de las copiosas nieves, que en este tiempo abundan en los montes y en la cordillera. Por ello, se forman nubes que perturban el equilibrio del aire, originando aquellos fortísimos vientos ${ }^{51}$.

Por último, Talavera recomienda que quien desee conocer más sobre los fenómenos del aire consulte las obras del Padre Gaspar Schott (1608-1666), Boyle, Nollet, Hauser y Berguer, pues allí se encontrarán noticias sobre máquinas, artefactos, fuentes neumáticas y otros instrumentos en uso; mientras que en particular sobre los vientos pueden consultarse en especial los trabajos de Musschenbroek y del ya mencionado Berguer ${ }^{52}$.

\section{Sobre el Agua}

El tercer capítulo del tratado se centra en el estudio del elemento agua (aqua). Aquí, los contenidos se estructuran en cuatro artículos, subdivididos a su vez en diversas cuestiones y secciones.

En el primer artículo, el preceptor aborda la distinción y diversidad del elemento agua, la que con justo mérito -dice- se distingue de los otros fluidos eternos conocidos, ya que es una substancia enteramente fluida, húmeda, inodora, transparente, incolora,

\footnotetext{
${ }^{49} \mathrm{Ibid}$., ff. 54r-56v.

${ }^{50}$ Ibid., f. $55 \mathrm{v}$.

${ }^{51}$ Ibid., f. 56v.

${ }^{52}$ Ibid., f. 56v.
} 
con módico calor volátil y un cierto grado de frío glacial, y que extingue por completo al fuego ${ }^{53}$.

Establece también una diferencia entre el agua pura y el agua natural: el agua pura es aquella en la que no se encuentran otras partículas heterogéneas, mientras que el agua natural es la que la naturaleza subministra para nuestro uso y que contiene diversas partículas heterogéneas, ya dañinas, ya salubres. El agua natural puede referirse, al parecer, a seis especies: pluvial (lluvia, nieve y granizos), de fuente (fontana), fluvial (fluviatilis), marina, de pozo (putealis) y de pantano o ciénaga (paludona) ${ }^{54}$.

Talavera, siguiendo las enseñanzas de Musschenbroek respecto a hechos observables en la naturaleza, destaca los abundantes efectos en el espíritu del agua de fuente de los campos de Toledo y Valencia. Por ello, relata que las aguas de sus fuentes tienen la virtud de transformar en piedra todos los cuerpos sumergidos en ellas, como lo escriben Vitrubio, Estrabón y Plinio, y también de infundir color a las crines, a los cabellos y a la lana. También se refiere brevemente a aquellas aguas «venenosas», con altos contenidos de arsénico o antimonio; y enseña las ideas de Kircher, expuestas en Mundo subterráneo, y las de Feijoo en el Teatro crítico universal, para referirse a las numerosas características del agua bebestible ${ }^{55}$.

En una cuestión exclusiva, estudia cuál es la naturaleza del agua y sus diversas propiedades. Primero, afirma que los físicos experimentales distinguen numerosas propiedades, entre ellas: la fluidez, la dureza y la volatilidad. Luego, expone cuatro conclusiones, entre cuyas ideas destacan, por ejemplo, aquellas sobre la compresión del agua de Francis Bacon, en el Novum Organum, y los experimentos físicos-mecánicos de Boyle. A modo de prueba, Talavera explica un experimento difundido por la Academia Florentina acerca de la compresión del agua, que dice fue probado por Musschenbroek y Nollet, según las enseñanzas de Almeida en Recreación filosófica (tomo III, vesp. 11ª). En este caso, la referencia a este último texto vuelve a ser exacta ${ }^{56}$.

En una cuestión distinta, Talavera analiza cuál es el origen del agua de las fuentes y de los ríos. Indica que sobre este punto los físicos asienten con unanimidad que su origen está en el mar. Sin embargo, discrepan si en forma mediata o inmediata. Así, de acuerdo con el preceptor existen tres opiniones: la cartesiana, que afirma que el agua marina se difunde en toda la masa de la Tierra y, por medio de canales ocultos en ella, llega hasta las raíces de los montes, en donde por proceso de percolación y diversos otros modos se transforman en dulces. La segunda, afirma que el origen de las fuentes debe ser renovado por las lluvias y la disolución de la nieve, las que, en especies de matraces subterráneos, son recolectadas en receptáculos preparados por la naturaleza, desde donde a través de pequeñas grietas surgen los efluentes, que descienden por las

\footnotetext{
${ }^{53}$ Ibid., f. 57 r.

${ }^{54}$ Ibid., f. 57r.

${ }^{55}$ Ibid., ff. 57v-58r.

${ }^{56}$ Ibid., f. 59r.
} 
pendientes de los montes hacia sus raíces, dando origen a las fuentes. Por fin, la última opinión distingue entre fuentes perennes y temporarias. Las primeras son aquellas que nunca decrecen, en cambio, las temporarias, aquellas que crecen cada cierto tiempo a causa de las tempestades y del derretimiento de las nieves ${ }^{57}$.

Luego, Talavera expone su propia visión de este fenómeno, afirmando que el origen de las fuentes y ríos perennes es el agua marina, la que se comunica en vapores sublimados o por medio de sifones subterráneos. Tal proposición la prueba con las enseñanzas de Kircher (Mundus subterraneus, II, 10; V, 2), según quien, en las profundísimas vísceras de la Tierra, especialmente en las cavernas de los montes, se hallan enormes lagos, fuentes y ríos perennes, cuyo origen es el agua marina.

En este punto se halla una de las características fundamentales del eclecticismo en la enseñanza de la filosofía de fines del siglo XVIII en las colonias iberoamericanas, presente también en la docencia de Manuel Antonio Talavera. Pues, las ideas aquí expuestas ${ }^{58}$ son probadas con dos pasajes de las Escrituras, a saber: Génesis $2,6^{59}$ y $10^{60}$. Sumado a ello, hay que señalar que el preceptor ofrece numerosos datos empíricos sobre el fenómeno particular en estudio, en este caso, las aguas de las fuentes y los ríos y su relación con el agua marina. La lista de autores referidos incluye, entre muchos otros, a Rigsiolus (Geographia, XVIII, 7), Edmundo Mariotte (1620-1684), Antonio Mayr (16731749), Claus Eduardo Corcini (1702-1765) y los ya mencionados Berguer, Almeida, Dechales, Boyle y Nollet ${ }^{61}$.

Por otra parte, Talavera aborda también el estudio acerca del mar o agua marina. Define, primero, algunos conceptos geográficos básicos relacionados con el tema, a saber: golfo, estrecho, canal, mar, archipiélago, cabo, isla, península, continente e istmo. Y, luego, caracteriza con detalle los distintos océanos y principales mares del planeta, sugiriendo revisar, a quienes deseen saber más, la Recreación filosófica de Teodoro de Almeida $^{62}$.

Respecto de dónde se origina la salinidad del mar (maris salcedo), el preceptor explica las ideas de Kircher, del Cardenal Ptolomeo, de Tomás Vicente Tosca (1651-1723), de Mayr y de Hauser, según las que proviene de las partículas salinas mezcladas en las aguas por el sapientísimo creador de todas las cosas. Al respecto, complementa sus enseñanzas con algunos testimonios expuestos en las Memorias de Trévoux (1723 y 1737) y en el Diccionario Physico de Mathurin Jacques Brisson (1723-1806). Son frecuentes también las referencias a célebres autoridades de la época, como el «Parisiensis Doctor et

${ }^{57}$ Ibid., ff. 62r-62v.

${ }^{58}$ Ibid., ff. 63r-63v.

${ }^{59}$ «Pero se levantaba de la tierra un vapor que regaba toda la superficie del suelo».

${ }^{60}$ «Y del Edén salía un río para regar el huerto, y de allí se dividía y se convertía en otros cuatro ríos».

${ }^{61}$ Talavera, Physica particularis, op. cit., ff. $63 \mathrm{v}-67 \mathrm{v}$.

${ }^{62}$ Ibid., ff. 67v-68v. 
celeberrimus medicus» Poisonier y la exposición detallada de las doctrinas físicas de Musschenbroek y Sigaud Lafond ${ }^{63}$.

Posteriormente, se ocupa de la causa de las mareas, sobre las que hay muchas opiniones -y también arrogancia, dice- entre los filósofos. Así, para algunos este tema es el sepulcro de la curiosidad humana; de acuerdo con otros, sólo profecías de los físicos y, para otros, el secreto del Creador. Comenta las explicaciones empíricas de este fenómeno brindadas por autores como el Padre Rigsiolus, Hauser, Berguer y Honorato Fabri (16071688), para referirse luego a la influencia de la luna sobre las mareas (véase la ilustración si-guiente) y explicar detalladamente las distintas fases del satélite natural. En este punto, el preceptor se vale también de numerosos datos entregados por los autores ya mencionados, además de los de Casinus (Actas de Ciencias Físicas de París, 1712, 1714 y 1720) ${ }^{64}$.

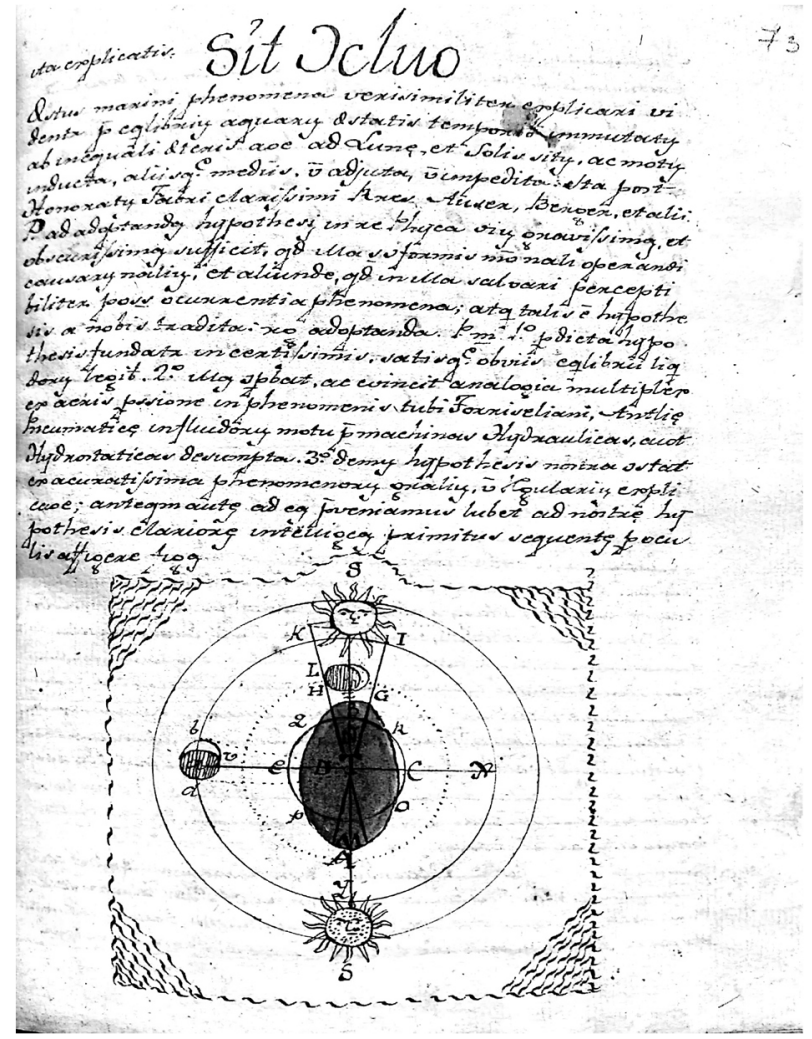

f. 73 r.

\footnotetext{
${ }^{63}$ Ibid., ff. $68 \mathrm{v}-71 \mathrm{r}$.

${ }^{64}$ Talavera, Physica particularis, op. cit., ff. 71r-74v.
} 
Más adelante, Talavera estudia las características y propiedades de las aguas minerales. Enseña que estas poseen propiedades que no se observan en el agua corriente, pues son derivadas de su mezcla con diferentes partículas de minerales, sales, piedras y metales. Siguiendo a Kircher, el preceptor distingue distintas especies de aguas minerales, entre ellas las mezcladas con diferentes materiales terrestres (aqua misceri), las salinas (salibus), las jugosas (succis) y las metálicas (metalicis corpusculis). Además, aborda los efectos en la salud de las aguas nocivas y comenta también los distintos efectos y usos de las aguas termales. Sobre esto último, entrega una serie de informaciones, recopiladas de las Memorias de Trévoux, acerca de las fuentes termales de Europa (principalmente Francia, Italia, Alemania y España) y de América. En este contexto, hallamos nuevamente su interés en explicar circunstancias locales, pues describe los múltiples beneficios de las termas de Potosí (Bolivia) y de las termas de Cauquenes (Chile) ${ }^{65}$.

Finalmente, en el último artículo de este capítulo, el preceptor explica los distintos meteoros del elemento agua. En este apartado, se refiere especialmente a los diferentes aspectos de los fenómenos compuestos por agua: la niebla (nebula), el sereno (aura serotina), el rocío (rore matutino), la escarcha (pruina), las nubes (nubibus), la lluvia (pluvia), la nieve (nive) y el granizo (grandine) ${ }^{66}$.

\section{Sobre la Tierra}

En el cuarto y último capítulo del tratado, Talavera estudia el elemento tierra (tellure). Desarrolla los contenidos en cuatro artículos y, en una sección especial, aborda extensamente el fenómeno de la electricidad.

En este punto hallamos una clara relación entre las materias abordadas y aquellas que son objeto de estudio de la llamada «Teoría de la Tierra». Pues, esta corriente fue, en efecto, un potente marco intelectual en el que se insertaron todas las investigaciones de las ciencias de la Tierra desde fines del XVII hasta comienzos del siglo XIX. En términos generales, consiste en una conjunción de la física cartesiana y de la Historia Sagrada, que brinda una explicación meramente teórica de la naturaleza de las cosas ${ }^{67}$.

En este contexto, hallamos que Talavera advierte que con el nombre de "Tierra» debe comprenderse, a la vez, al globo terráqueo junto con las aguas que contiene, dispuestas de esa forma -y como todo en ella- por la divina sabiduría de Dios. Por ello, constituye un objeto dignísimo de la contemplación física. En esta línea, indica que

${ }^{65}$ Ibid., ff. $77 \mathrm{v}-79 \mathrm{r}$.

${ }^{66}$ Ibid., ff. 79r-83r.

${ }^{67}$ Cf. Roger, J., «La théorie de la Terre au XVIIe siècle», Revue d'histoire des sciences, 26/1 (1973), pp. 23-48, en p. 23. Según este autor, la expresión «Teoría de la Tierra» aparece en orden cronológico en las siguientes obras: Telluris Theoria Sacra de Burnet (1681); A new Theory of the Earth de William Whiston (1696); Théorie de la Terre de Buffon (1749), Theory of the Earth de Hutton (1795). Un discípulo de éste, John Playfair, escribe Illustrations of the Huttonian Theory of the Earth (1802). Por último, el término también aparece en Discours sur les révolutions du globe de Cuvier (1812). 
Aristóteles definía a la tierra como un elemento seco y gravífico, fundamento de todos los sabores en tanto vehículo, mientras que los Recentiores lo definen como un elemento firme, seco y sumamente opaco. En todo caso, el preceptor insiste en que la tierra por la que caminamos no es verdaderamente el elemento, sino que, como múltiples experimentos lo prueban, las distintas especies de tierras son, más bien, compuestos ${ }^{68}$.

En un primer artículo, Talavera se refiere a la figura natural (physica) de la Tierra. Indica que, sobre esto, son numerosas las opiniones de los antiguos. Así, para Anaximandro, la Tierra tenía forma columnar; según Anaxímenes, era plana; para Leucipo también era plana, pero como un pandero (tympano); y según Demócrito, era cóncava como un disco. Mientras que -dice- para Tales, Parménides, Platón y Aristóteles tenía la forma de un globo. Luego, revisa los argumentos que afirman que la masa terráquea tiene una figura redonda o esférica. En este punto, para probar su argumentación se sirve ampliamente de la evidencia empírica expuesta por el Padre Tosca y comenta también algunas curiosas disputas en la Iglesia acerca de la existencia de las Antípodas ${ }^{69}$.

Más adelante, el preceptor se refiere a un tema muy controvertido en los estudios físicos: la figura matemática de la Tierra. En efecto, al respecto muchas son las opiniones de los antiguos y de los modernos y, también, las disciplinas involucradas para la investigación de este tema, como la geografía, la astronomía y la náutica, según enseña Hauser en su Physica particularis. De acuerdo con Talavera, los matemáticos modernos, especialmente los franceses e ingleses, consideraban que la Tierra no era matemáticamente redonda, sino, más bien, esférica. Para probar esta idea, revisa extensamente argumentos de diversa índole (geométricos, trigonométricos y astronómicos) e incluye también una ilustración para explicar con claridad los contenidos. Aquí, en general, se vale de las obras de Hauser, Musschenbroek y de las actas de la Academia de París (1718, 1737 y 1739) y presenta numerosos datos sobre experimentos de medición de la Tierra, para concluir su forma esferoidal, achatada en los polos y alargada en el ecuador ${ }^{70}$.

Por otro lado, Talavera se refiere al estudio de la magnitud de la Tierra, la que puede considerarse doblemente. Por un lado, la magnitud relativa, que considera a la Tierra comparada con el cielo estrellado y, por otro, la magnitud absoluta, que considera a la Tierra en sí misma, por medio de sus medidas propias. Indica que, de acuerdo con Rigsiolus, el globo terráqueo es semejante a un punto en relación con el cielo estrellado, lo que es coherente con la sabiduría común de todas las opiniones. Y esta misma idea dice Talavera- es la que expresaba Séneca al escribir: «Hoc est illud punctum quod inter tot gentes ferro et ignem divitur? O quam parvuli [ridiculi] sunt mortalium termini!» (Naturales Quaestiones, Praefatio, I, 9) ${ }^{71}$.

${ }^{68}$ Talavera, Physica particularis, op. cit., f. 83v.

${ }^{69} \mathrm{Ibid}$., ff. 83r-85v. Se refiere en particular a la carta del Papa San Zacarías ante la denuncia de San Bonificio de que Virgilio enseñaba la existencia de las Antípodas.

${ }^{70}$ Ibid., ff. $85 \mathrm{v}-90 \mathrm{r}$.

${ }^{71}$ Ibid., f. 90v. 
No obstante, el preceptor insiste en la importancia de estudiar sobre este tema, pues, aún siendo un punto, es sobre la Tierra que viajamos a través del espacio. Por ello, siguiendo al Padre Tosca, se refiere a las ideas de los geógrafos modernos con respecto a la periferia de la Tierra o circunferencia ecuatorial, pues a partir de ello derivan numerosos experimentos para calcular el diámetro del planeta.

Otro punto al que el preceptor atiende es sobre la constitución o cara exterior de la Tierra. En este contexto, explica qué se entiende por valles, planicies y colinas, y pone especial cuidado en estudiar el origen de los montes. Señala que, principalmente, los montes más altos fueron formados en el origen del mundo por la suprema sabiduría de Dios, pues, de acuerdo con las Escrituras, ya existían antes del diluvio universal. Por ello, advierte, en muchos montes se hallan tanto restos de conchas ${ }^{72}$, peces y otros muchos cuerpos marinos, como también de elementos vegetales incrustados en las piedras. En la misma línea, explica qué son los fósiles y detalla numerosos experimentos, principalmente los de Georges Baglivi (1669-1707) en De vegetatione lapidum (1714) y de Tournefort en las Memorias Regiae Scientiarum Academiae (1700 y 1702). Luego, Talavera dedica un extenso apartado exclusivo para referirse a aquellos cuerpos marinos que están en la mayoría de los montes ${ }^{73}$. Sobre este punto en particular, resulta de importancia destacar la semejanza en la elaboración de los contenidos estudiados por Talavera con la corriente intelectual de los «Teóricos de la Tierra» que ya hemos mencionado ${ }^{74}$.

Más adelante, en el cuarto y último artículo de este capítulo, se dedica a estudiar la estructura interior de la Tierra. Siguiendo el modelo kircheriano (Mundus subterraneus, II, 19), propone que, a semejanza de los miembros del cuerpo humano, el cuerpo de la Tierra está equipado con muchos talleres (officinarum), que producen variados humores y licores hacia el interior y el exterior de ella. Estos talleres subterráneos son de tres géneros: hidrofilacio, pirofilacio y aerofilacio. No obstante, añade que en la masa de la Tierra se hallan cuerpos de varios otros géneros, como las piedras, los imanes, las resinas, los metales y semimetales, las sales, los jugos oleosos y la tierra, y todos ellos se funden en el reino mineral ${ }^{75}$. Por esto, siguiendo con la estructura metodológica del tratado, el preceptor estudia cada uno de aquellos elementos en parágrafos especiales.

De este modo, en primer lugar se refiere brevemente a las diversas teorías sobre el origen de las piedras (influencias celestes, semillas petrificadas y el jugo lapidificador) y aborda con detalle las diferentes cualidades de las piedras comunes, de las piedras marmóreas y de las gemas, indicando recurrentemente como medio de prueba las obras de Baglivi, Tournefort, Feijoo, Hauser, Sigaud Lafond y Kircher ${ }^{76}$. Luego, explica las virtudes del imán, al que define como una piedra negruzca y ferruginosa que se halla en

${ }^{72}$ Éstas, según William Whiston en su obra A new Theory of the Earth (1696), serían medallas del diluvio universal. Cf. Roger, «La théorie de la Terre au XVIIe siècle», op. cit., p. 46.

${ }^{73}$ Talavera, Physica particularis, op. cit., ff. 91r-93r.

${ }^{74}$ Cf. Roger, «La théorie de la Terre au XVIIe siècle», op. cit., pp. 46-48.

${ }^{75}$ Talavera, Physica particularis, op. cit., f. 93v.

${ }^{76}$ Ibid., ff. 93v-96v. 
las minas de hierro, cuyas principales propiedades son la atracción, repulsión, comunicación, dirección, declinación e inclinación. Sobre este punto, en general, Talavera se sirve nuevamente de los testimonios de Dechales, Tosca, Berger, Hauser y Nollet ${ }^{77}$. Dedica, además, una amena «advertencia médica», con referencias exactas a las fuentes, para referirse a las virtudes del imán en el tratamiento y alivio de los dolores de dientes y en las palpitaciones del corazón. Por último, explica acerca de las resinas (bituminibus) y los jugos oleosos o sulfúricos (succis oleosis) e indica que son todos los cuerpos densos y viscosos, como los aceites que expulsan animales y plantas. Distingue, a su vez, entre resinas fluidas y sólidas y expone distintas opiniones sobre el ámbar, a la que define como una substancia densa fragantísima e inflamable ${ }^{78}$.

Por otra parte, en una sección especial, aborda también el fenómeno de la fuerza eléctrica en tres cuestiones exclusivas. En este punto, Talavera declara seguir las hipótesis más cercanas a la verdad, expuestas principalmente por Nollet, De Lana, Fabri y Benito Navarro en su Physica electrica (1752) ${ }^{79}$. Primero, el preceptor explica en qué consiste esta fuerza y se refiere a algunos de los fenómenos eléctricos ígneos. Y, luego, explica la comunicación y la propagación de la fuerza eléctrica, siguiendo expre-samente las obras de Musschenbroek, Hauser, Feijoo y Sigaud Lafond, además de las de los autores antes nombrados ${ }^{80}$.

Con posterioridad, enseña brevemente acerca del origen y las diversas propiedades de los metales y semimetales (oro, plata, cobre, hierro, estaño, plomo y mercurio). Aquí, el preceptor destaca el error de los Peripatéticos al estimar que cada planeta producía un metal en particular. Además, explica brevemente las características y propiedades de las sales nativas o naturales (salibus nativis) y los jugos minerales (succis mineralibus) ${ }^{81}$.

Finalmente, en el último artículo del tratado, Talavera se refiere a la tierra (terra), advirtiendo que, con este concepto, no entenderá aquí el elemento, sino los cuerpos compuestos por otros elementos, como la tierra fósil, que contiene partículas ligeramente unidas, secas, opacas y esponjosas, que rociada con agua se convierte en una masa húmeda; o aquellas tierras que presentan diversos colores y texturas. No obstante, se dedicará a estudiar con mayor detención, en una cuestión única, cuál es la causa de los terremotos.

Al respecto, refiere que, por una parte, Aristóteles (Meteora, II, 2) ya distinguía dos géneros de terremotos: las sacudidas (tremorae), con movimiento horizontal, y el pulso (pulsus), con movimientos verticales más frecuentes; $y$, por otra, Séneca, quien también reconocía varias especies del fenómeno telúrico. Agrega luego que para los filósofos gentiles, principalmente los babilonios y asirios, los terremotos tenían origen en la

\footnotetext{
${ }^{77}$ Ibid., ff. 96v-99v.

${ }^{78}$ Ibid., ff. $99 \mathrm{v}-100 \mathrm{r}$.

${ }^{79}$ Ibid., f. 100v.

${ }^{80}$ Ibid., ff. 102v-106v.

${ }^{81}$ Ibid., ff. 106v-108v.
} 
influencia de los planetas y que, después, su causa se atribuyó a la acción de los dioses. En cambio, de acuerdo con los Recentiores, son varias las causas de los terremotos: la más común afirma que se producen por una vehemente rarefacción del aire con la asistencia de materias sulfúreas inflamadas o fuegos subterráneos ascendentes; según otros, se producen por la compresión del aire, siendo suficiente para ello únicamente la rarefacción; mientras que, para otros, la trepidación de la tierra no puede causarse sino por medio de las fuerzas eléctricas. Esta última opinión es la que a juicio de Manuel Antonio Talavera es la más probable, siendo sostenida principalmente por Feijoo (tomo V epistolarum, carta 28), de quien entrega también la referencia exacta ${ }^{82}$.

\section{Conclusiones}

El Tratado acerca de los elementos es el primero de los contenidos en la Física particular, que, a su vez, es la segunda parte de las Instituciones de Física de Manuel Antonio Talavera. La Física particular incluye, además, otros dos tratados: Acerca de los seres vivos (De viventibus, ff. 112r-179r) y Acerca de los cuerpos celestes (De coelo et Mundo, ff. 179r-212v). Por desgracia, el curso sobre la Física general -primera parte de las Instituciones-, a la que el autor hace numerosas referencias a lo largo del texto, se encuentra actualmente perdido.

El Tratado acerca de los elementos revisa principalmente las doctrinas físicas según las enseñanzas de los filósofos modernos (Recentiores). En él hallamos expuestas detalladamente tanto las doctrinas de los filósofos clásicos como de los físicos modernos sobre los elementos fuego, aire, agua y tierra, que son los constitutivos de los cuerpos naturales.

A partir de los contenidos aquí revisados por Talavera, queda en evidencia que era conocedor de la obra de los más importantes físicos modernos, en especial de Newton y Boyle, principalmente -según él mismo lo consigna con frecuencia- a través de los eruditos compendios de Hauser, Musschenbroek y Feijoo, y de las célebres Memorias de Trévoux.

En este sentido, las doctrinas físicas expuestas por el preceptor son, en general, fundamentadas en la experiencia y en el testimonio y la autoridad de las fuentes citadas. De modo que, además de las numerosas referencias a los Recentiores, encontramos, en menor medida, el recurso a la fundamentación de acuerdo con las Escrituras. No obstante, la obra entrega igualmente valiosa información respecto de las fuentes utilizadas para la enseñanza de la filosofía en aquella época, como, por ejemplo, los diferentes compendios que ya hemos mencionado o las Actas de las distintas Academias Científicas. Asimismo, vale la pena resaltar el intento de Talavera de complementar las opiniones

\footnotetext{
${ }^{82}$ Ibid., ff. 108v-111r.
} 
físicas de los modernos con interpretaciones científicas propias sobre diferentes fenómenos naturales observados en el Chile de la época.

De acuerdo con lo anterior, el valor de este curso radica en ser una fuente primaria íntegra, de puño y letra del propio autor, en la que se halla el rico testimonio de la enseñanza de la filosofía natural en los últimos tiempos de la Colonia. Como hemos intentado mostrar, en la obra se plasma una muy particular manera de concebir y explicar los fenómenos naturales, recurriendo para ello tanto a diferentes explicaciones teóricas e históricas y a la autoridad de las Escrituras como también a numerosa evidencia empírica. Se aprecia así una concepción de la física más cercana a la ciencia experimental, alejada paulatinamente del enfoque más conceptual-metafísico de los pensadores escolásticos de la modernidad temprana. En este sentido, el curso de Talavera es un claro ejemplo del período que Celina Lértora Mendoza denomina preilustrado ecléctico, una de las etapas propias de la escolástica colonial americana, en la que se evidencia un conocimiento ya propio de la modernidad europea, matizado con elementos característicos de la escolástica tradicional.

En consecuencia, con este trabajo creemos haber hecho un aporte doble. Pues, por un lado, hemos rescatado una parte importante del legado filosófico latinoamericano, lo que permitirá nuevas investigaciones en esta área de la disciplina, y, por otro lado, haber hecho visible, al menos en una mínima parte, el quehacer filosófico de Manuel Antonio Talavera, profesor particular de la disciplina en el Chile de la última época colonial.

Abel Aravena Zamora

abel.aravena@upla.cl

Fecha de recepción: 04/04/2019

Fecha de aceptación: 01/07/2019 